\title{
Inclusive Fitness does not Impact the Survival Processing Effect
}

\author{
Mark A. Krause ${ }^{1 *}$, Shaina Trevino ${ }^{2}$, Andrea Cripps ${ }^{1}$, Katie Chilton ${ }^{1}$, \\ Emma Sower ${ }^{1}$, and John P. Taylor ${ }^{1}$
}

\author{
${ }^{1}$ Department of Psychology, Southern Oregon University \\ ${ }^{2}$ Prevention Science Institute, University of Oregon \\ *Corresponding author (Email: krausema@sou.edu)
}

Citation - Krause, M. A., Trevino, S., Cripps, A., Chilton, K., Sower, E., \& Taylor, J. P. (2019). Inclusive fitness does not impact the survival processing effect. Animal Behavior and Cognition, 6(1), 13-31. https://doi.org/10.26451/abc.06.01.02.2019

\begin{abstract}
Natural selection acts upon learning and memory processes, much as it does with other physical and behavioral characteristics. For example, numerous lines of inquiry using nonhuman species hypothesize adaptive specializations of Pavlovian conditioned responses and spatial memory. Comparatively less is known about the influence of evolution on learning and memory processes in humans, but the past decade has seen a growing interest in this area; particularly since the publication of Nairne, Thompson, and Pandeirada's (2007) paper reporting the survival processing effect (SPE). The SPE describes a mnemonic advantage of considering one's survival in an evolutionarily relevant scenario: Words encoded in such a scenario are better remembered than are words encoded in relation to survival irrelevant scenarios. Numerous publications inspired by Nairne et al.'s paper have subsequently attempted to describe the specific mechanisms, evolutionary and otherwise, that account for the SPE. In four experiments we tested whether manipulating levels of inclusive fitness in survival scenarios influences recall. In our scenarios we asked participants to consider the survival of kin, and other categories of social relationships (self, strangers, family, a famous person), in an evolutionarily relevant context. The basic SPE was replicated in three of the four experiments. However, although kin selection is an important evolutionary mechanism, it appears to be unrelated to the SPE in our sample.
\end{abstract}

Keywords - Survival processing effect, Kin selection, Inclusive fitness, Adaptive memory, Evolution of learning, Evolution of memory

Behavioral phenotypes evolve in fitness-enhancing directions. Even learning and memory processes, which enhance adaptive behavior within the lifetime of the individual, are influenced by natural selection. For instance, Pavlovian conditioning is a general mechanism of associative learning, but the nature of the types of associations that organisms form reflects responses to ecological pressures (Hollis, 1997; Krause \& Domjan, 2017). Fear-evoking stimuli such as snakes and spiders are particularly salient at a perceptual level, and investigators have argued that many species of primates are predisposed to rapidly learn about their threat potential (Isbell, 2006; Öhman \& Mineka, 2001). Interspecific variation in behavior and hippocampal anatomy among seed-caching corvids may reflect different selection pressures on spatial memory (Gould et al., 2013). Learning also directly impacts reproductive success. Learning about cues that predict the availability of a mate results in increased reproductive success in Japanese quail, Coturnix japonica (Domjan, Mahometa, \& Matthews, 2012), and learning about cues that predict the approach of predators improves survival in prairie dogs, Cynomys ludovicianus (Shier \& 
Owings, 2006). The comparative psychological literature is rich with examples of interactions among learning, memory, and evolution.

One way that comparative psychologists conduct experiments on the adaptive significance of learning and memory is to use ecologically relevant stimuli within the experimental design and context, and contrast conditioning to such naturalistic stimuli with arbitrary or ecologically neutral stimuli (Domjan, Cusato, \& Krause, 2004). Experiments designed like this can answer questions about how learning and memory processes are evolutionarily adaptive (e.g., ultimate level explanations), and build upon the large literature that is predominantly focused on proximate level mechanisms.

A similar approach can, in principle, be used to study human memory. However, the literature on human learning and memory, namely declarative memory, has not been driven by questions about evolution to the extent seen in comparative psychology. Perhaps the strength of natural selection on human learning and memory processes is relaxed in comparison to that of other species, and therefore its evolutionary signatures are not as readily decipherable. Alternatively, there may simply be less contemporary interest in evolutionary questions about human learning and memory (though this would not characterize historical trends, see Boakes, 1984). One notable exception can be found in the body of literature examining the survival processing effect (SPE) originally described by Nairne et al. (2007). Here, words encoded in relation to survival in an evolutionarily relevant scenario (e.g., what comparative psychologists might call "naturalistic") are better remembered in comparison to words encoded in relation to non-evolutionary scenarios (e.g., "arbitrary"). A standard methodology for studying this effect requires participants to first read a scenario asking them to imagine they are in a grassland environment thought to resemble ancestral habitat conditions wherein they must find resources and avoid predators. By comparison, another group is asked to imagine that they are moving to a new country and must prepare for all of the uncertainty that this entails. Participants then rate items on an identical list of words for their relevance to the corresponding scenario. After a distractor task (e.g., digit span task) a surprise memory test for the rated words is given. Nairne et al. reported superior memory for words in the survival scenario compared to words in the moving scenario, as well as in comparison to a control condition requiring participants to simply rate whether the same words were pleasant or unpleasant. To date the SPE has proven to be a robust one, as demonstrated by meta-analysis of survival processing studies using various iterations of this procedure (Scofield, Buchanan, \& Kostic, 2018).

The decade that has followed Nairne et al.'s (2007) description of the SPE has seen a growing literature testing different hypotheses relating to the core question of whether evolutionary processes account for the mnemonic advantage of remembering in the context of survival demands (e.g., Nairne \& Pandeirada, 2016; Schwartz, Howe, Toglia, \& Otgaar, 2014). Some investigators question whether an evolutionary interpretation is required to account for the SPE. For example, Soderstrom and McCabe (2011) report that survival in various contexts, not just an ancestral one, provides mnemonic advantage. Klein, Robertson, and Delton $(2010,2011)$ showed that imagining oneself in the future improves recall, regardless of whether that imagined future entails considering one's survival. The types of hypotheses tested in studies examining the SPE parallel those found in the literature on animal learning and memory. There is not a consensus that certain types of Pavlovian conditioned responses, spatial memory, and other commonly cited examples truly reflect adaptive specializations of learning and memory in nonhumans (Domjan \& Galef, 1983; Krause, 2015a). For example, Öhman and Mineka (2001) hypothesize an adaptive "fear module" that accounts for the primate predisposition to acquire fear responses toward snakes. However, this may not be an adaptive specialization of learning, per se, but rather an outcome of enhanced perception and attention toward snake or snake-like objects (Isbell, 2006; Krause, 2015a). Thus, a proximate level explanation may provide a more parsimonious account of the ultimate one.

Relatedly, there are at least a dozen proximate mechanisms that have been advanced to try to explain the SPE (Erdfelder \& Kroneisen, 2014; Krause, 2015b). A specialized "survival module," in evolutionary psychology terms, is among them. But many of these proximate mechanisms appear to have little direct relevance to evolutionary processes (see Nairne \& Pandeirada, 2016, for discussion). However, evolution is not driven by a single factor or mechanism. Survival is a necessary but not sufficient condition for evolutionary change-differential reproductive success among individuals 
expressing alternative, heritable phenotypes are essential components, and myriad factors influence reproductive success. Mate choice, jealousy, guarding, parental care, cheater detection, and avoiding incest are also outcomes and processes driving evolutionary change, and, hence, the nature of physical and behavioral adaptations. Sandry, Trafimow, Marks, and Rice (2013) used several alternative fitnessrelevant scenarios (e.g., jealousy, mate selection, infidelity) in their study of the SPE. Memory for words encoded in response to the standard survival scenario was superior to these evolutionary alternatives. This speaks to the robust nature of the SPE but is puzzling with regard to proposing evolutionary explanations to explain it. Calling something a survival module does not adequately explain the multitude of processes, evolutionary and otherwise, accounting for superior recall in evolutionary contexts. Thus, we can further understand the nature of what is adaptive about adaptive memory by testing whether there is a mnemonic advantage to remembering in contexts that are related to other influential evolutionary processes.

One such possibility is kin selection. In addition to acting on individuals, natural selection acts on groups of close relatives. Social animals that live in close spatiotemporal proximity to genetic relatives can directly or indirectly influence each other's reproductive success. Organisms that help their genetic relatives, even at a cost to themselves, will be favored by evolution if their actions increase net genetic fitness (Hamilton, 1964). This is a very general summary of how altruistic behavior can evolve, although satisfactory empirical support for Hamilton's rule may be wanting (see Nowack, McAvoy, Allen, \& Wilson, 2017). For example, natural selection can influence altruism only if the specific genes that underlie altruistic behaviors are selected for, and this is actually quantified far less than kin selection is invoked. Still, humans are among the most cooperative of all animal species, and typically favor the wellbeing of genetic relatives over non-relatives and unfamiliar individuals. Much of the experimental literature on human cooperation is done with participants who are unrelated to each other; in part because cooperation between unrelated individuals is more interesting than between genetic relatives (Rand \& Nowak, 2013), and we might add that study samples typically involve undergraduate students with low coefficients of relatedness to each other. Regardless, humans' long history of favoring kin over non-kin when it comes to cooperating, protecting and helping should have signature effects on cognitive traits, possibly including memory.

Investigators of the SPE have included socially relevant contexts into their scenarios. For example, Kostic, McFarlan, and Cleary (2012) tested whether being alone or with a group has any relative mnemonic advantage (it does not). Studies examining the role of self-referential processing in the SPE have included scenarios in which participants contemplate either their own survival or the survival of another person (Cunningham, Brady-Van den Bos, Gill, \& Turk, 2013). To our knowledge, previous investigations of the SPE have not incorporated scenarios in which participants must explicitly consider the survival of related and unrelated group members.

In four experiments we manipulated the composition of social groups included in modified versions of the scenarios originally used by Nairne et al. (2007). If kin selection, and specifically the survival of kin, is a component of the survival module hypothesized to underlie the SPE, then more words should be remembered when kin are specified in those scenarios relative to non-kin. Furthermore, encoding scenarios that involve survival processing should result in superior memory for words compared to a survival irrelevant control condition. The degree to which kin are made salient and focal to the survival scenarios was manipulated across Experiments 1-3. Experiment 4 was conducted to test whether people who are close to us (e.g., family, friends) matter more in a survival processing context relative to an individual who is completely unrelated and affiliated with participants.

\section{Experiment 1}

\section{Method}

Participants were 207 undergraduate students enrolled in psychology courses at Southern Oregon University. They received a small amount of extra credit for their participation. We conducted the 
experiment in classrooms using computer and projection equipment to present instructions, prompts, and experimental stimuli.

Participants were randomly assigned to one of three experimental conditions or to a control condition. In the experimental conditions, three different scenarios were presented in which we asked participants to consider their survival in an ancestral environment (identical to Nairne et al., 2007, in this regard), but included revised text instructing them to consider the survival of kin (group KIN, $n=54$ ), unrelated group members (NON-KIN, $n=58$ ), and the self (SELF, $n=42$ ). We included a control group $(n=53)$ that did not read a survival scenario but viewed and rated the same words for their pleasantness (see Table 1 for scenarios). After providing consent, each participant was given a packet including the study materials. We tested in classrooms to expedite the data collection process, which is a method used by other investigators conducting similar studies (e.g., Klein et al., 2010). In our own experiments on the SPE we have found very similar effects when testing participants either individually or with testing in classroom settings (Haskett, Skou, Krause, \& Taylor, 2014; Taylor et al., 2013).

Table 1

Scenarios Read prior to Encoding Task for each Group in Experiment 1 Condition and Scenario

SELF: In this task we would like you to imagine that you are stranded in the grasslands of a foreign land, without any basic survival materials. Over the next few months, you'll need to find steady supplies of food and water and protect yourself from predators. We are going to show you a list of words, and we would like you to rate how relevant each of these words would be for you in this survival situation. Some of the words may be relevant and others may not-it's up to you to decide.

KIN: In this task we would like you to imagine that you and your family (including relatives and your own children) are stranded in the grasslands of a foreign land, without any basic survival materials. Over the next few months, you will need to find steady supplies of food and water and protect your family from predators. We are going to show you a list of words, and we would like you to rate how relevant each of these words would be for you and your family in this survival situation. Some of the words may be relevant and others may not - it is up to you to decide.

NON-KIN: In this task we would like you to imagine that you and several unrelated people with whom you live with are stranded in the grassland of a foreign land, without any survival materials. Over the next few months, you will need to find steady supplies of food and water and protect your group from predators. We are going to show you a list of words, and we would like you to rate how relevant each of these words would be for you and your group in this survival situation. Some of the words may be relevant and others may not - it is up to you to decide.

CONTROL: In this task, we are going to show you a list of words, and we would like you to rate the pleasantness of each word. Some of the words may be pleasant and others may not - it is up to you to decide.

Note: The SELF and CONTROL conditions are identical to the survival and pleasantness ratings conditions from Nairne et al. (2007). The NON-KIN and KIN scenarios are modified to prompt participants to consider the survival of relatives (KIN) or nonrelatives (NON-KIN) during the word rating task.

The experiment began when participants turned the cover page and read their respective scenarios (Table 1) printed on the next page. After reading the scenarios and instructions, participants were prompted to turn the page and prepare to rate 32 individually presented words for their relevance (or pleasantness) to the scenario just read. Ratings ranged on a 1 (totally irrelevant) to 5 (extremely relevant) scale for the three survival scenarios, and a 1 (totally unpleasant) to 5 (extremely pleasant) scale for the control condition. Each word was presented for $10 \mathrm{~s}$, giving participants ample time to view and rate it. After the word rating task participants were given a digit span memory task in which six sets of ten digits were presented in succession. Participants were asked to view each digit set (presented at a rate of $2 \mathrm{~s}$ per digit) and write down as many they could remember immediately afterward. Following the digit span task, which lasted about $5 \mathrm{~min}$, participants were instructed to turn the page, which prompted them to write down as many words as they could remember from the 32-item list they had previously rated. They were given 6 min to attempt to recall and write as many words as possible. 
To ensure compliance with the study protocol, students were instructed to turn to a subsequent page only when prompted by the experimenter. This was especially important because the memory test at the end of the experiment needed to be unexpected. In addition to the experimenter, one or two assistants (depending on the size of the class) ensured compliance with procedures. A concern with group testing was that participants would attempt to compare words they wrote down during the final word memory test (despite having no extrinsic incentive to do so). Data from one participant who failed to comply with instructions was discarded.

\section{Results and Discussion}

On average, more words were remembered in each of the three survival conditions in comparison to the control group [KIN: $M(S E)=21.4(0.57)$, NON-KIN: 20.9 (0.55), SELF: $20.3(0.65)$, CONTROL: $18.3(0.58)]$. Univariate ANOVA revealed a significant main effect for the four groups, $F(3,203)=5.61$, $p=.001, \eta_{p}{ }^{2}=.77$; see Figure 1A). The greater number of words remembered in the three survival conditions compared to the control group confirms that survival processing provides a mnemonic advantage. However, total words remembered did not significantly differ among any of the three groups that read survival-based scenarios (pairwise comparisons were $p=.203$ and higher for all three group comparisons).

Average word ratings were compared across groups to test whether words were processed differently based on the scenarios read. This is important to explore because group differences in words remembered could possibly be explained by congruity effects, rather than survival processing per se. An ANOVA on word ratings across the four scenario groups showed no main effect, $F(3,203)=2.32, p=$ $.077, \eta_{p}{ }^{2}=.033$. For exploratory purposes we conducted pairwise comparisons on the four groups and found that participants given the SELF scenario had a significantly lower average word rating than did groups with any of the other three scenarios ( $p<.05$ for the three pairwise comparisons; see Figure 1B). No other pairwise comparisons were significant. Although word congruity has been shown to contribute to the SPE (Palmore, Garcia, Bacon, Johnson, \& Keleman, 2012), similarly to Nairne et al. (2007) we did not find evidence for word congruity effects.

To test whether word ratings were related to memory we computed difference scores on word ratings by subtracting, for each subject, their average rating for words they forgot from the average rating of words that were remembered. Using these difference scores as a dependent variable, we found a significant main effect for group, $F(3,203)=8.63, p<.001, \eta_{p}{ }^{2}=.11$ (Figure 1C). Pairwise comparisons showed that all three survival groups were more likely to remember words rated as more relevant to their respective scenarios in comparison to words rated as less relevant, and participants in the control group remembered more words that they rated as unpleasant than pleasant. No significant differences were found among the KIN, NON-KIN, and SELF groups for difference scores on ratings for remembered versus forgotten words (all $p \mathrm{~s}>$.759). All three experimental groups had significantly higher difference scores in comparison to the control group (all $p$ s $<.001$ ). These results should be interpreted with caution since the difference scores are a quasi-experimental variable, and the ratings scale was not identically applied by the control group in comparison to the experimental groups.

The inclusion of either kin or non-kin individuals in the scenarios had no effect on recall. One possible reason is that the wording that specified the presence of family or non-relatives within the scenarios was not sufficiently salient to engage the participants' attention. Perhaps the most salient feature of the scenario involves avoiding predators or securing vital resources, and considering survival of group members, related or not, does not affect encoding or retrieval. 
Krause et al. 18

A)

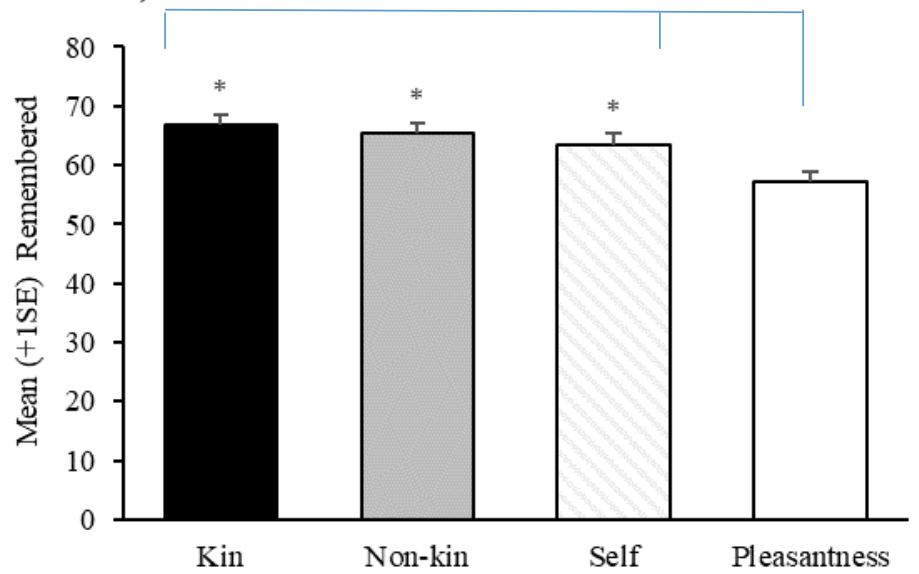

B)
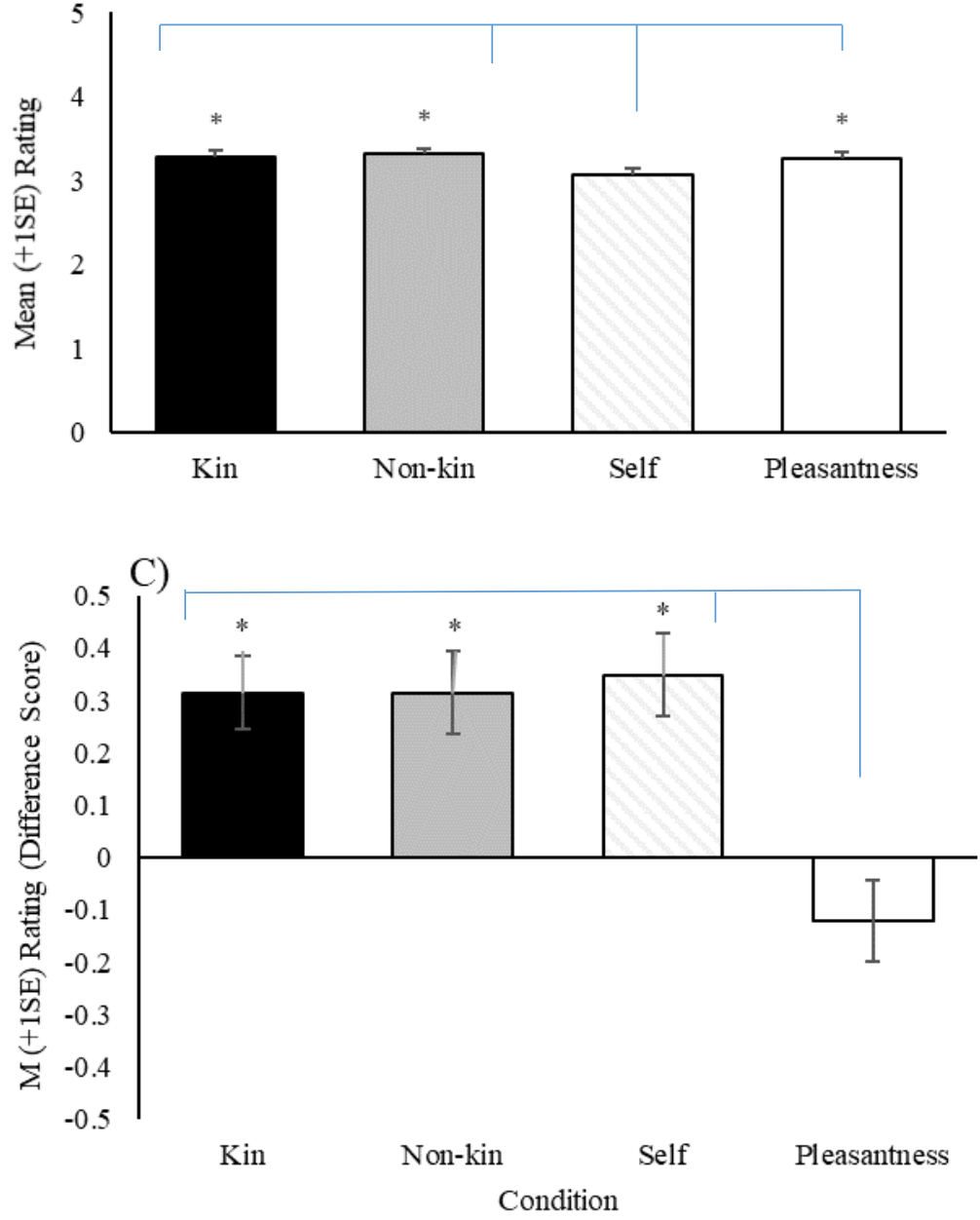

Figure 1. Mean (+1SE) words remembered for each condition (A), ratings (B), and difference scores for ratings on words remembered minus forgotten $(\mathbf{C})$ in Experiment $1 . *$ indicates a significant difference $(p<.05)$ in post-hoc tests for those conditions compared to the condition without $*$. 


\section{Experiment 2}

We designed Experiment 2 to test whether isolating the effect of social relatedness of group members in a survival scenario would lend support to the kin selection hypothesis of the SPE. We created a new set of scenarios in which references to avoiding predators and securing food and water were removed. The scenarios still involved promoting survival, but details on the context or specific tasks required were not given. Kroneisen and Erdfelder (2011) found that reducing the tasks within the survival scenario (e.g., just focusing on finding potable water) attenuates recall, and, thus, the SPE. They suggest that it is the richness in which words are encoded that provides the mnemonic advantage. However, humans are highly responsive to social context, and simplifying the scenarios such that participants pay closer attention to the social dynamic of the scenario provides a possibly more direct way of testing whether inclusive fitness is relevant to the SPE.

\section{Method}

Participants were 121 undergraduate students enrolled in psychology courses at Southern Oregon University. They received a small amount of extra credit for their participation. As with Experiment 1 we ran the experiment in classrooms using computer and projection equipment to present instructions, prompts, and experimental stimuli. Data from two participants were discarded because they failed to comply with instructions.

The basic procedure and design were identical to that of Experiment 1 . We included the same four groups: KIN $(n=30)$, NON-KIN $(n=31)$, SELF $(n=32)$, and CONTROL $(n=29)$. The modified scenarios, with references to avoiding predators and securing resources removed, are shown in Table 2.

Table 2

Scenarios Read prior to Encoding Task for each Group in Experiment 2 Condition and Scenario

SELF: In this task, we would like you to imagine that your survival and well-being is threatened. You find yourself in a position where you alone must defend yourself. We are going to show you a list of words, and we would like you to rate how relevant each of these words would be for you in this survival situation. Some of the words may be relevant and others may not - it's up to you to decide.

KIN: In this task, we would like you to imagine that the survival and well-being of your close family members is threatened. You find yourself in a position where you alone must defend them. We are going to show you a list of words, and we would like you to rate how relevant each of these words would be for defending your family in this survival situation. Some of the words may be relevant and others may not - it's up to you to decide.

NON-KIN: In this task, we would like you to imagine that the survival and well-being of several people who are unfamiliar to you is threatened. You find yourself in a position where you alone must defend them. We are going to show you a list of words, and we would like you to rate how relevant each of these words would be for defending these people in this survival situation. Some of the words may be relevant and others may not - it's up to you to decide.

CONTROL: In this task, we are going to show you a list of words, and we would like you to rate the pleasantness of each word. Some of the words may be pleasant and others may not - it is up to you to decide.

\section{Results and Discussion}

Similar to Experiment 1, more words were remembered in each of the three survival conditions in comparison to the control group [KIN: $M(S E)=16.5(0.79)$, NON-KIN: $16.2(0.78)$, SELF: $17.4(0.76)$, CONTROL: $13.9(0.80)$ ]. Univariate ANOVA revealed a significant main effect of scenario, $F(3,118)=$ $3.55, p<.05, \eta_{p}{ }^{2}=.08$ (see Figure 2A). The significant main effect is attributed to superior recall for words in all the three experimental group scenarios compared to the pleasantness group (all $p \mathrm{~s}<.05$ in 
post-hoc comparisons). However, none of the three experimental groups significantly differed in recall (all $p s>.24)$.
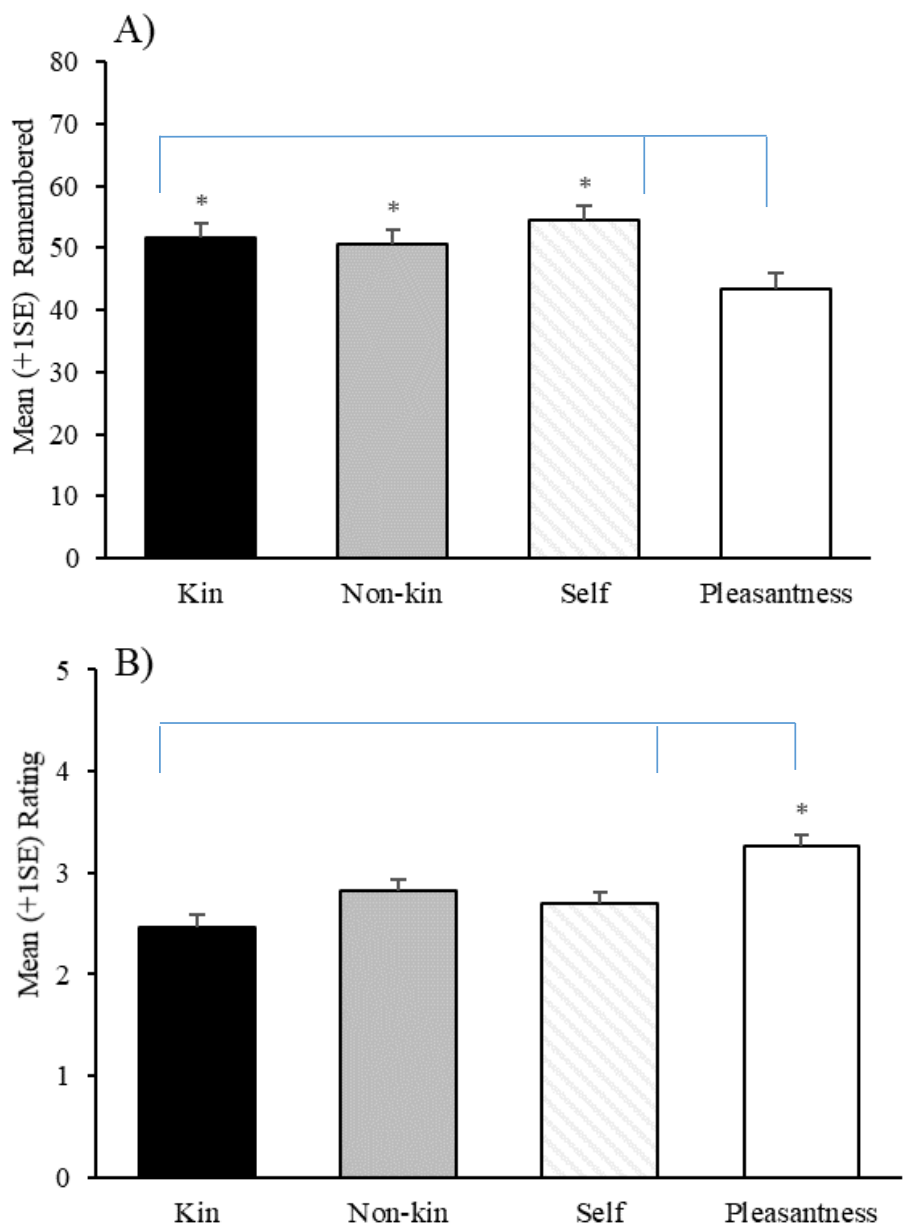

C)

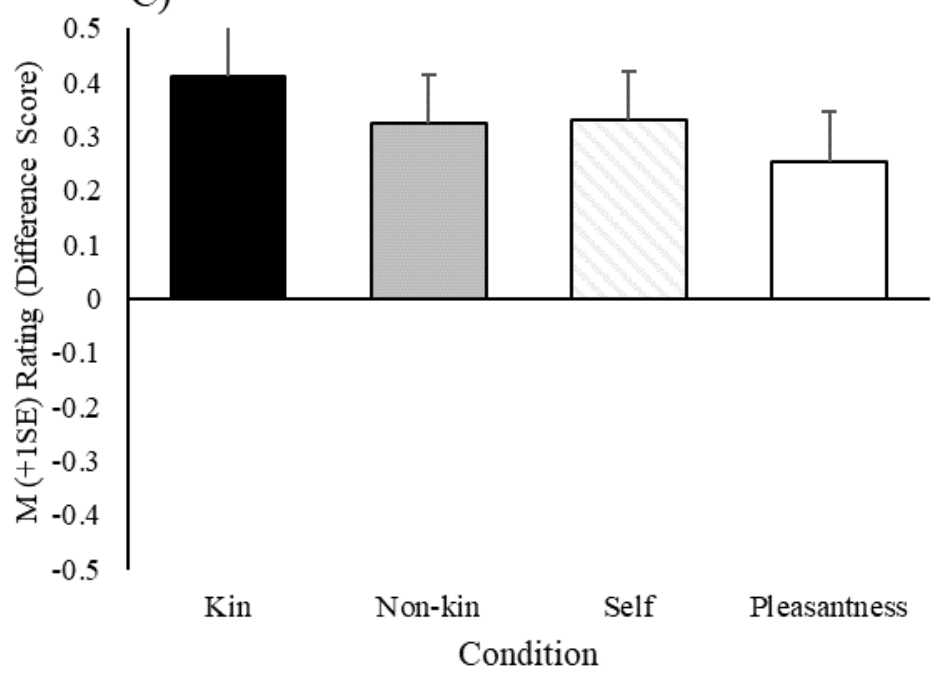

Figure 2. Mean (1SE) words remembered for each condition (A), ratings (B), and difference scores for ratings on words remembered minus forgotten $(\mathbf{C})$ in Experiment $2 . *$ indicates a significant difference $(p<.05)$ in post-hoc test for those conditions compared to the condition without $*$. 
We found a significant main effect of group on word ratings, $F(3,117)=8.49, p<.001, \eta_{p}{ }^{2}=.18$ (Figure 2B). On average, participants gave higher ratings to words in the pleasantness CONTROL condition in comparison to all three experimental groups (post-hoc comparisons for all $p \mathrm{~s}<.01$ ). The KIN group gave lower overall ratings to words in comparison to the NON-KIN group $(p=.025)$. The KIN and SELF and NON-KIN and SELF groups did not significantly differ in their mean word ratings (all $p \mathrm{~s}>$.144). Mean word ratings were unrelated to whether they were subsequently remembered or forgotten, as no main effect was found for difference scores on ratings for words remembered versus forgotten, $F(3,118)=0.487, p=.692, \eta_{\mathrm{p}}^{2}=.012$ (Figure 2C).

As with Experiment 1 we did not find evidence to suggest that the presence of relatives in a survival scenario influenced recall ability. Removing other survival relevant features in the scenario (finding resources and avoiding predators) such that participants might focus on the social aspects of the scenarios did not influence recall in Experiment 2 (see Kroneisen \& Erdfelder, 2011, for contrasting results). Alternatively, priming participants to think concretely about the social components of the scenarios prior to encoding and rating words might increase the salient feature of the survival of other individuals, and thus testing whether kin selection is relevant to the survival processing effect.

\section{Experiment 3}

In Experiment 3 we attempted to increase the degree to which kin or non-kin were relevant to the scenarios by first having participants write about a personally relevant interaction with a related or unrelated person prior to reading the scenarios used in Experiment 2. The rationale for this procedure was based on results showing that writing about an emotional event influences mood states (Mills \& D'Mello, 2014), which in turn should increase how participants view the salience of social relatedness while reading survival scenarios.

\section{Method}

Participants were 203 undergraduate students enrolled in psychology courses at Southern Oregon University. They received a small amount of extra credit for their participation. As with Experiments 1 and 2, data were collected in classrooms using computer and projection equipment to present instructions, prompts, and experimental stimuli. Participants were randomly assigned to KIN $(n=53)$, NON-KIN $(n=$ 51), SELF $(n=47)$, and CONTROL $(n=52)$ groups. Data from four participants were discarded because they failed to comply with instructions. We followed the same basic procedures of Experiments 1 and 2 (reading scenarios, rating words, digit span, memory test), except that prior to reading the survival scenarios participants were instructed to spend five minutes writing about either a relative (KIN), an unrelated person (NON-KIN), themselves (SELF), or about an object (CONTROL; see Table 3 for the wording of the writing prompts). The KIN, NON-KIN and KIN groups read survival-based scenarios and the CONTROL group completed the word pleasantness rating task following the writing prompt phase.

Table 3

Paragraph Writing Prompts used in Experiment 3

Condition and Instructions

Self: Write a paragraph describing an activity you recently engaged in.

Kin: Think of a specific, living family member with whom you currently have a close relationship. Write a paragraph describing your most recent interaction with this person.

Non-Kin: Think of a specific, living person (non-relative) whom you know casually and neither like nor dislike. Write a paragraph describing your most recent interaction with this person.

Control: Write a paragraph describing an object that you currently own.

Note: Scenarios read following the paragraph writing task were identical to those used in Experiment 2. 


\section{Results and Discussion}

Word recall was similar across the KIN $[M(S E)=15.2(0.69)]$, NON-KIN: $15.4(0.7)$ and CONTROL [15.0 (0.69)] groups, and the SELF scenario group remembered, on average, more words $[17.0(0.73)]$. However, the main effect of scenario was not significant, $F(3,199)=1.65, p=.179, \eta_{p}{ }^{2}=$ .024 (Figure 3A). Post-hoc tests revealed that the SELF group remembered significantly more words than did the CONTROL group $(p<.05)$.
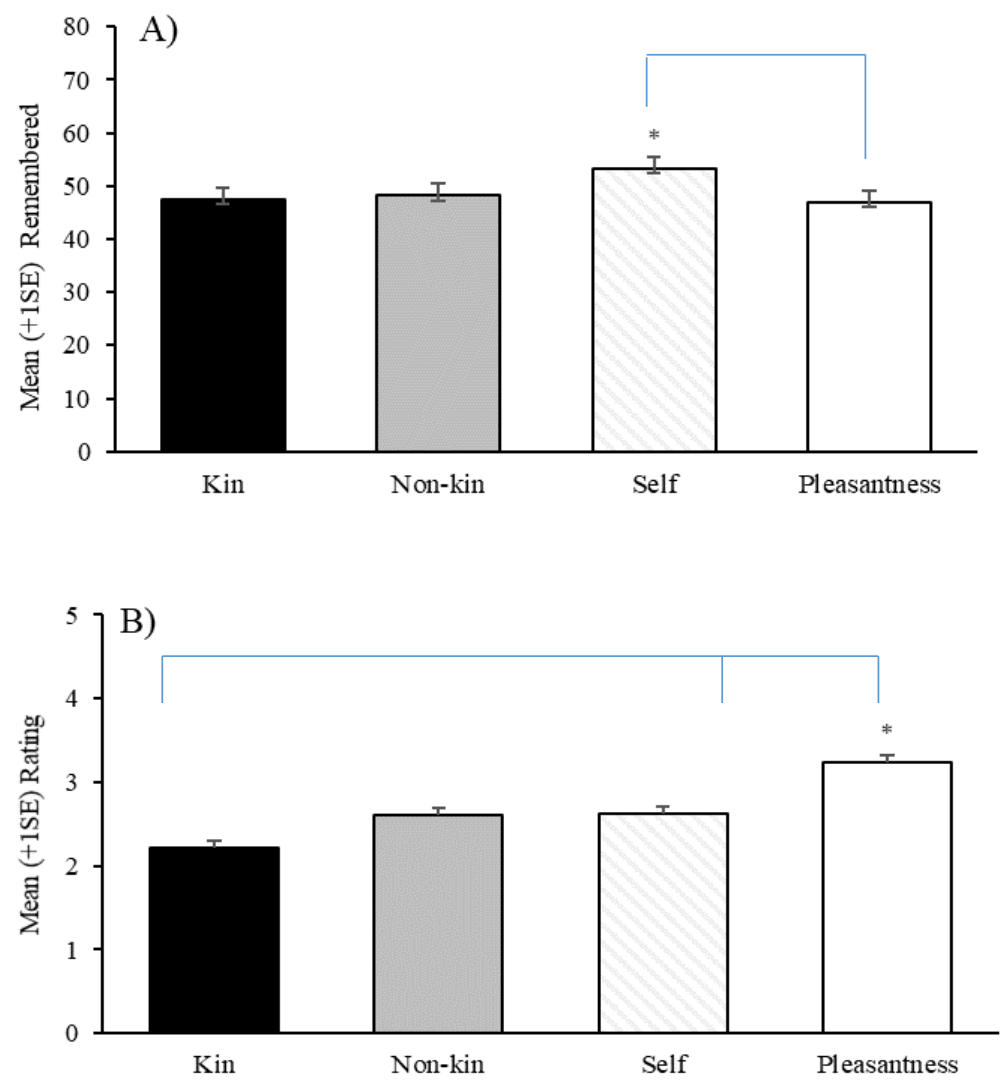

C)

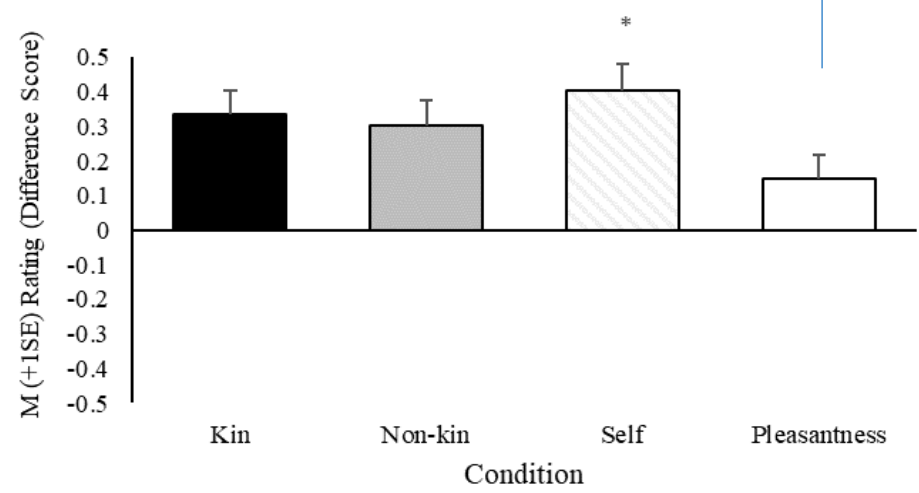

Figure 3. Mean (1SE) words remembered for each condition (A), ratings (B), and difference scores for ratings on words remembered minus forgotten $(\mathbf{C})$ in Experiment 3. * indicates a significant difference $(p<.05)$ in post-hoc test for those conditions compared to the condition without $*$. 
Words ratings differed, on average, among the four groups, $F(3,199)=25.27, p<.001, \eta_{p}{ }^{2}=$ .276 (Figure 3B). The pleasantness group (CONTROL) had higher average word ratings than all three experimental scenarios (all $p s<.001$ in pairwise comparisons). The NON-KIN and SELF groups had higher average word ratings compared with the KIN group $(p s<.01)$. Overall, there was no main effect on ratings for remembered versus forgotten words, $F(3,199)=2.14, p=.096, \eta_{p}{ }^{2}=.031$ (Figure 3C). Post-hoc tests showed that mean ratings for words remembered were significantly higher for the SELF group in comparison to the pleasantness ratings for the CONTROL group $(p<.05)$.

We found no evidence that inclusive fitness influences how people respond to survival relevant scenarios in this experiment. Furthermore, the SPE was not nearly as robust in this experiment as observed in Experiments 1 and 2. This was unexpected given how consistently the SPE occurs across multiple laboratories (e.g., Kang, McDermott, \& Cohen, 2008; Müller \& Renkewitz, 2015; Schwartz et al., 2014). Greater word recall in the SELF group in comparison to CONTROL should be interpreted carefully, of course, as the overall main effect of group was not significant. Previous work has shown that there is a mnemonic advantage for self-referential processing (Klein, 2012; Symons \& Johnson, 1997), which may also account for our findings in Experiment 3.

Results from Experiments 1 and 2 indicate that other people, kin or non-kin, matter just as much as oneself when it comes to survival processing, as indicated by similar levels of recall across KIN, NONKIN, and SELF groups. Our hypotheses have focused on testing the degree to which relatedness matters but results thus far suggest that the SPE is unrelated to the category or specific identity of the individual(s) whose survival is threatened. However, each of the preceding experiments either left it up to the participants to decide the specific identity of the group members involved (KIN and NON-KIN in Experiments 1 and 2) or were asked to decide on a specific individual (Experiment 3). There remains a possibility that the NON-KIN scenarios used in Experiments 1-3 did not sufficiently establish that the unrelated people are not somehow directly connected to the participants (e.g., they imagined strangers who were at least familiar on some level, such as other classmates). This makes it difficult to determine whether any of the survival scenarios used in the experiments reported thus far have tested whether it is actually survival or a sort of "extended" self-reference influencing the results. In this case, self-reference would encompass both the participant in the experiment directly (e.g., in the SELF condition of Experiment 1) or more broadly to include people who are within the social sphere of the participant (KIN, NON-KIN groups). Including a fully unrelated but recognizable individual is one way to test this possibility.

\section{Experiment 4}

Experiment 4 was designed to test whether people included in survival scenarios need to be closely associated to the participants, and in what way, to boost recall relative to a control condition. Being "closely associated" could be interpreted as anything from people who are unfamiliar but nearby in terms of spatial proximity, to our nearest genetic relatives. We altered the scenarios in two ways to reduce ambiguity in how participants interpret the social relatedness of the individuals included in them. First, experiments 1 and 2 did not direct participants to imagine specific individuals (e.g., a sibling or parent) while reading the scenarios. Doing this for dozens of participants, though possible, is not necessarily feasible. We used a practical alternative in Experiment 4 by asking participants in the KIN condition to imagine that their youngest blood relative was involved in the survival scenario. This helped ensure that a specific genetic relative was identified, and that their survival was considered during the encoding phase. Second, we included a condition that involved someone who is a familiar public figure but presumably not socially related to the participants in any way. Cunningham et al. (2013) used this approach in their study testing the effect of self-referential encoding on the SPE. Participants imagined themselves in the standard SELF condition (e.g., same as Experiment 1 of this study and Nairne et al., 2007). In their "other" condition the participants were asked to imagine then British Prime Minister David Cameron as the individual in the survival scenario. Experiment 4 was not designed to test for self-referential processing, but the idea of using a single, familiar but unrelated individual was germane to our question 
of whether imagining people who are close to the participant in some way (e.g., a specific family member or friend) leads to deeper encoding and increased recall relative to someone completely unrelated, as well as to a control condition. ${ }^{1}$

\section{Method}

Participants were 167 undergraduate students enrolled in psychology classes and an introductory biology class at Southern Oregon University. As with Experiments 1-3 data were collected in classrooms using computer and projection equipment to present instructions, prompts, and experimental stimuli. Participants were randomly assigned to KIN $(n=36)$, FRIEND $(n=44)$, FAMOUS $(n=42)$, and CONTROL $(n=45)$ groups. Data from ten participants were discarded because they failed to comply with instructions. We followed the same basic procedures of Experiments 1 and 2 (reading scenarios, rating words, digit span, memory test).

The scenarios were the same as used in Experiment 1 except for the content of the first sentence. The control condition (word pleasantness rating) was identical to that of Experiments 1 and 2. For the KIN group, the first sentence read: In this task we would like you to imagine that your youngest blood relative is stranded in the grasslands of a foreign land, without any basic survival materials. We specified "youngest blood relative" to ensure that, compared to other conditions, a genetically related family member was chosen, and specifying that the person be the youngest was done to increase the chance that the (presumed) viability of each individual might be factored into the choice. For the FRIEND group the first sentence read: In this task we would like you to imagine that a friend (who is not a member of your family) is stranded in the grasslands of a foreign land, without any basic survival materials. For the FAMOUS group, the first sentence read: In this task we would like you to imagine that singer Ariana Grande is stranded in the grasslands of a foreign land, without any basic survival materials. We selected a celebrity who would likely be at least recognizable by name by the majority of the participants. At the end of the experiment participants were asked to write down the specific relative or friend they chose, and to identify whether they knew that Ariana Grande is a celebrity. Five of the 10 participants who were dropped from the study had been assigned to the KIN group (original $n=41$ ) and were removed because they failed to identify a blood relative. Three of the 42 participants in the FAMOUS condition did not recognize Ariana Grande. However, these participants were not dropped because the scenario implies that she is famous, which would be sufficient to encourage imagining someone completely unrelated during the encoding task. The criteria for dropping or keeping participants in the KIN and FAMOUS groups were established prior to conducting any analyses.

\section{Results and Discussion}

Word recall was higher among the experimental groups relative to the control group [KIN: $M$ $(S E)=17.3$ (0.74), FRIEND: 19.1 (0.67), FAMOUS: 18.9 (0.69), CONTROL: 14.9 (0.66)], and the overall main effect was significant, $F(3,163)=8.44, p<.001, \eta_{p}{ }^{2}=.134$ (Figure 4A). Post-hoc comparisons showed that all three experimental groups remembered significantly more words than did the control group (all $p s<.05$ ). There were no significant differences in recall among the three experimental groups (all $p s>.08)$.

We found a main effect for word ratings, $F(3,163)=7.94, p<.001, \eta_{p}{ }^{2}=.128$, which was due to the significantly higher ratings in the CONTROL group relative to the KIN, FRIEND, and FAMOUS groups ( $p<.01$ for all three comparisons, Figure 4B). Average word ratings did not differ significantly among the three experimental groups (all $p s>.16$ ). ANOVA on difference scores subtracting ratings on words forgotten from words that were remembered was significant, $F(3,163)=3.74, p<.05, \eta_{p}{ }^{2}=.064$. This relatively weak effect was due to significantly lower difference scores for the CONTROL group

\footnotetext{
${ }^{1}$ Experiment 4 was recommended by an anonymous reviewer.
} 
relative to all experimental groups (all $p s<.05$, Figure 4C), which in turn did not differ from each other in their ratings difference scores (all $p s>.623$ ).

A)

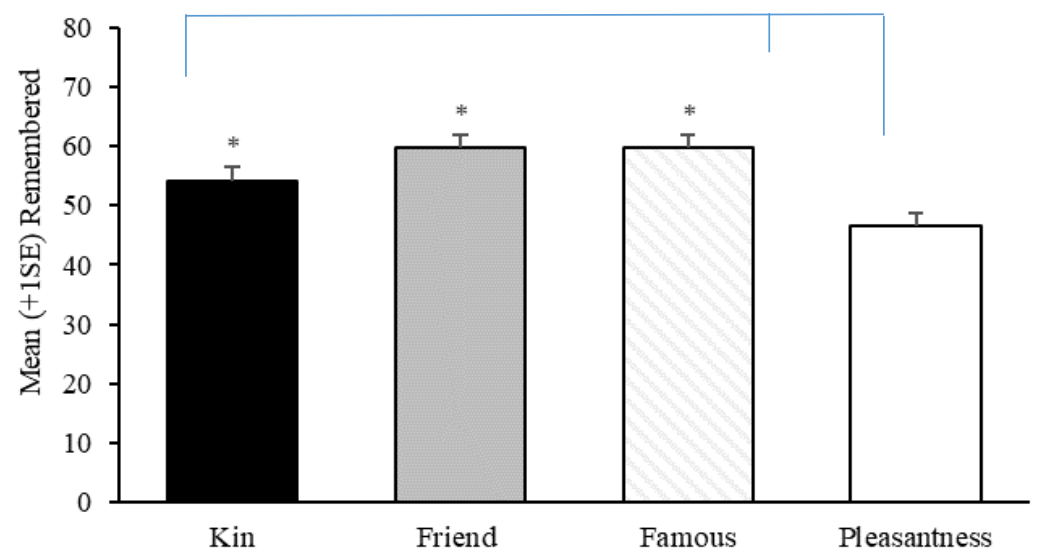

B)
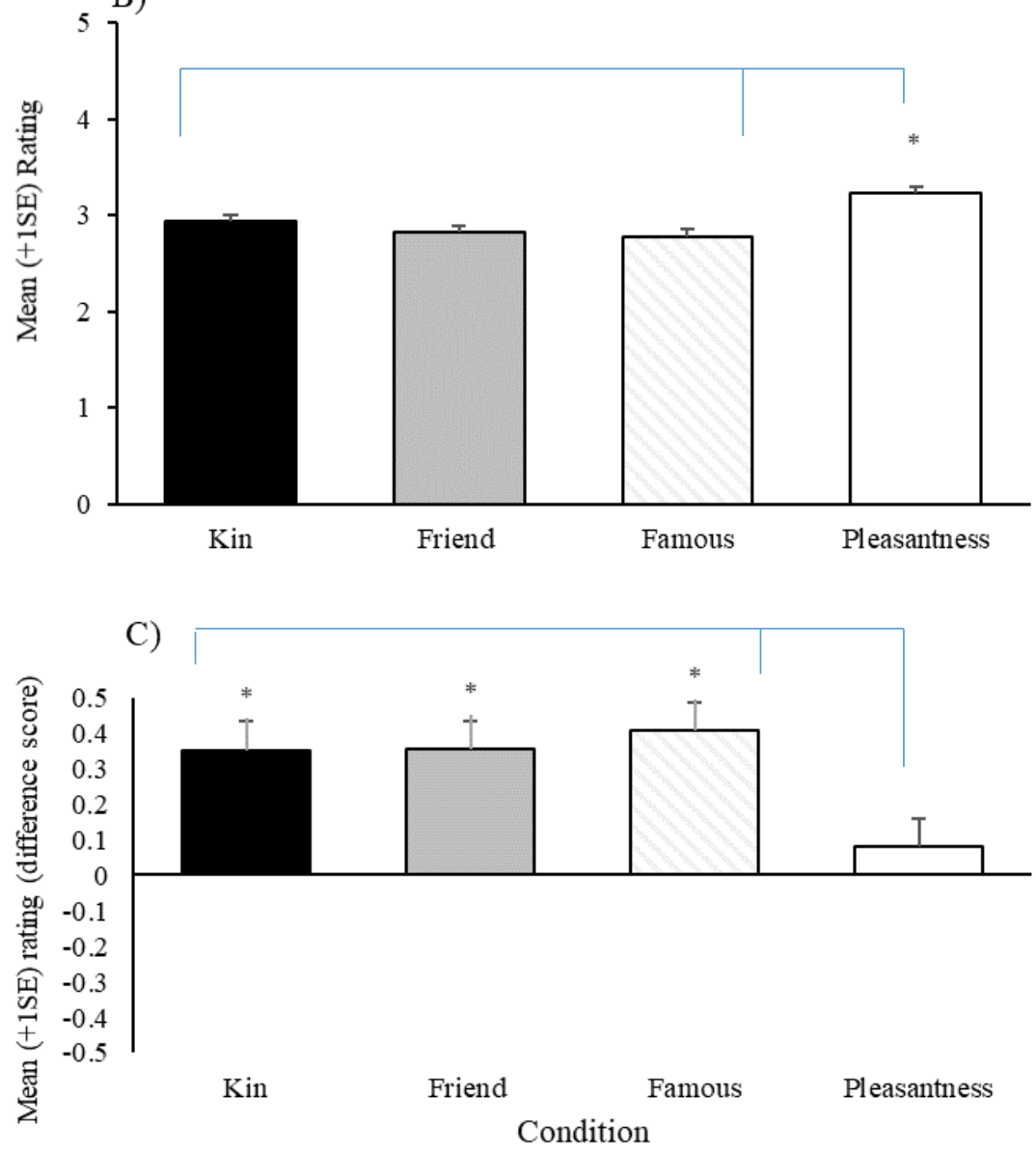

Figure 4. Mean (1SE) words remembered for each condition (A), ratings (B), and difference scores for ratings on words remembered minus forgotten $(\mathbf{C})$ in Experiment $4 . *$ indicates a significant difference $(p<.05)$ in post-hoc test for those conditions compared to the condition without *. 
Similar recall in performance in the KIN and FRIEND groups aligns with the results of Experiments 1 and 2, which showed consistency in how the presence of others during encoding, regardless of degree of relatedness, influences survival processing. However, word recall in the FAMOUS group was similar to that of the KIN and FRIEND groups, indicating that the SPE occurs even when someone very removed from our direct social sphere (e.g., is not a "close" relation in any sense of the word) is included in the encoding scenario. This contrasts with the results of Cunningham et al. (2013), who found that word recognition was reduced in the group that imagined the survival of David Cameron in comparison to the group that contemplated their own survival. With regards to Experiment 4, imagining that a famous person is seeking shelter, finding water, and avoiding predators is indeed a novel and salient task that might only serve to strengthen encoding to a degree similar to that of imagining a close friend or relative, if not for different reasons.

\section{General Discussion}

In the past ten years, investigators have tested numerous hypotheses probing the evolutionary significance of the SPE. Some have focused on determining whether basic established memory phenomena account for it (see Howe \& Otgaar, 2013; Schwartz et al., 2014) while others have sought to understand the nature of its adaptive and evolutionary bases (Nairne \& Pandeirada, 2008, 2016). The present study is consistent with the latter approach, though we do not take it as given that an evolutionary perspective is presently required to fully account for the SPE. We used a novel approach to test whether kin selection would offer increased mnemonic advantage. We replicated the survival processing effect in Experiments 1, 2, and 4 as indicated by superior recall among groups that read survival scenarios compared with controls. However, asking participants to consider the survival of others, including kin, held no relative mnemonic advantage in any of the four experiments we conducted.

Some considerations for future work include increasing the length of word lists and manipulating the type of kin or non-kin relations that the participants are asked to consider in the scenarios (e.g., one's own offspring, specific siblings, childhood friend). The similar levels of word recall in the three experimental groups in Experiments 1, 2, and 4 could be due to a ceiling effect that is specific to the word list used, and if relative differences among the groups were to occur, then longer, or perhaps different, lists could confirm this possibility. Also, survival is but one of many factors that motivates our concern for kin. An alternative would be to examine whether considering kin in scenarios involving altruism or enhancing reproductive success would yield mnemonic advantage relative to non-kin and control scenarios.

It could be argued that the results from Experiments 1, 2, and 4 show a scenario effect in relation to the pleasantness control condition, but not a survival effect because non-survival scenarios were not included. The current set of experiments were specifically designed to test the kin selection hypothesis. That we did not get these results could suggest that, in hindsight, a non-survival scenario would have added to ongoing discussion about whether survival processing is different from contextually rich but survival irrelevant scenarios. However, similar to Sandry et al. (2013, Experiments 1 and 2) our interest was in the relative differences among fitness related scenarios, rather than fitness versus non-fitness related ones.

Kin selection is commonly invoked by social scientists to explain altruistic behavior in humans, but not always with the level of accuracy and operational specificity as used in evolutionary biology (Park, 2007). Kin selection would certainly seem to account for why people favor genetic relatives over nonrelatives. However, invoking kin selection as the causal process by which a behavioral pattern evolves does not discount other valid alternatives. For example, cultural factors may reinforce the same favoritism toward relatives as predicted by kin selection (see Archer, 2013, for examples involving family violence). Also, cultural mechanisms may themselves contribute to kin selection, as preferential treatment towards those with whom we spend the most time with may stem from factors of socialization that are fitness enhancing. The current study may not have been adequately designed to isolate an effect of kin selection on memory, if indeed there is such an effect. Furthermore, as Hamilton (1964) stated, inclusive fitness is 
relevant only to genes that underlie altruistic behavior, not the proportion of shared DNA per se. Regarding this point, our failure to detect significant differences in survival processing between the KIN and NON-KIN groups may also be attributed to a negligible influence of kin selection on heritable variation in episodic memory ability.

\section{Fitness-enhancement and Memory}

Numerous psychological processes are fitness enhancing, and those that promote survival are among them, but fitness is also enhanced by finding and securing mates, maintaining status within one's social group, and detecting deception or cheating. If selection has fine-tuned the manner in which human episodic memory is used, then it can be hypothesized that memory for fitness-relevant information would be evidenced in other fitness-enhancing contexts. Sandry et al. (2013) compared recall performance across multiple fitness-relevant scenarios, including fear/phobia, cheater detection, mate selection, incest avoidance, jealousy, infidelity, status, and, critically, the survival scenario used by Nairne et al. (2007). Superior recall was found for the survival condition relative to each of the other fitness-relevant scenarios, as well as two control conditions.

The results of Sandry et al. (2013) demonstrate the robust nature of the SPE; but it is also rather puzzling that individual survival trumps other fitness-relevant processes that, from an evolutionary perspective, are equally if not more relevant to lifetime reproductive success. An individual that is prolific in terms of successful reproduction but experiences a premature death because he or she failed to avoid a predator or find food and water, is still highly successful from a Darwinian perspective. The present study adds kin selection to the list of fitness-relevant scenarios that appear either unrelated or irrelevant to the SPE. One notable difference between Sandry et al. and the present study is that we found that recall was similar across survival conditions (KIN, NON-KIN, and SELF), whereas Sandry et al. report superior recall in their survival condition relative to other experimental conditions. This may have occurred because we made relatively minor modifications to the original scenarios of Nairne et al. (2007) to add the element of social relationships and relatedness, and Sandry et al. created novel scenarios for each fitness-relevant condition. Thus, while the SPE is robust in relation to the parameters of one scenario and its minor variants, it does not seem to generalize across scenarios with rich evolutionary significance. This was the case even when we attempted to enhance the salience of the survival of other people (KIN vs NON-KIN) in Experiments 2 and 3.

\section{Domain General or Domain Specific Processing?}

Our failure to find any effect of kin on the SPE, as well as results of studies discussed previously, suggests that contemplating survival in a general, if somewhat ambiguous evolutionary context, is all that is required to elicit the SPE. This conclusion is consistent with previous work showing that the survival processing effect occurs in response to unlikely, or even impossible, scenarios. For example, the survival advantage generalizes across contexts including desert, jungle, lost-at-sea, and lost-in-outer space scenarios (Kostic et al., 2012). Thus, although survival is one among several variables that contribute to evolutionary success, the survival advantage as it pertains to memory processing may be domain-general, rather than a narrow, fine-tuned cognitive adaptation (Soderstrom \& Cleary, 2014; see also meta-analysis by Scofield et al., 2018).

The social context of the scenarios used in research examining the SPE is worthy of further consideration. The standard grasslands survival scenario (e.g., Nairne et al., 2007; the "self" condition in Experiment 1 of the current study) involves isolation from others. Kostic et al. (2012) tested whether social isolation is the key variable underlying the SPE by comparing scenarios in which the participants imagined themselves lost at sea either alone versus among a group of other people. Recall was similar in both alone and group conditions, indicating that social isolation is not the salient feature driving the SPE. Our results from experiments 1 and 2 are consistent with those of Kostic et al. in that we found no 
differences in scenarios involving groups of people (KIN and NON-KIN) in comparison to the SELF condition.

A noteworthy result is that people's faces are better remembered if they were previously considered in the context of their potential as a long-term mating partner, as opposed to a long-term working relationship (Pandeirada, Fernandes, Vasconcelos, \& Nairne, 2017). Using faces instead of words as stimuli in survival processing experiments is an ecologically relevant approach. Facial recognition is an important capacity for socially complex animals and has been demonstrated across a broad range of species, including nonhuman primates (Parr, 2011). However, Savine, Scullin, and Roediger (2011) found that survival processing does not generally seem to benefit memory for faces to the extent that it does for words. This includes situations in which the identity of the face is linked to kinrelevant attributes such as being family-oriented and physically healthy (Savine et al., 2011, Experiment 5). Negative evidence such as this may delineate boundary conditions of the SPE. It may be that the mnemonic benefit of survival processing is constrained and limited to rather specific situations and stimulus types, rather than generalizable across many relevant sources of information that would be valuable to remember (see Nairne \& Pandeirada, 2016, for discussion).

\section{The Evolution of Animal and Human Learning and Memory}

The theoretical debates about the SPE in humans are reminiscent of ones found in the animal learning literature. For example, the somewhat interchangeable concepts of preparedness and adaptive specializations of learning developed in the late 1960s following Garcia and Koelling's (1966) groundbreaking work on conditioned taste aversions. Debates ensued over whether conditioned taste aversions (and other phenomena) represented "prepared" or adaptively specialized learning, or, alternatively, could be explained by established, general process learning principles (Domjan \& Galef, 1983; Logue, 1979). Despite slow progress toward confirming the validity of concepts such as preparedness and adaptive specializations of learning (Domjan \& Galef, 1983), evidence using established methods for testing evolutionary adaptations has grown (Krause, 2015a). For example, studies of Pavlovian conditioning of quail (Coturnix japonica) have demonstrated a direct relationship between conditioning and reproductive success (Domjan et al., 2012), and experimental evolutionary studies with Drosophila have mirrored the natural contexts in which learning capacities (e.g., preparedness) might evolve (Dunlap \& Stephens, 2014).

Parallel debates in the human memory literature have played out among those who view the SPE as either a form of adaptive memory, or an effect that can be accounted for by proximate memory processes that do not require an evolution-based layer of interpretation (Kazanas \& Altarriba, 2015; Krause, 2015b; Kroneisen \& Erdfelder, 2011; Nairne \& Pandeirada, 2016). For example, Kroneisen and Erdfelder (2011) maintain that survival per se is not the key element that provides mnemonic advantage during encoding, but rather the richness and distinctiveness of the information presented. Specifically, when the standard survival scenario is reduced to a single element (e.g., finding potable water), the SPE is significantly reduced. Similarly, Klein (2012) concluded that self-referential processing has an important mediating influencing the SPE. These are just two examples - a specialized "survival module" is among a dozen different proximate mechanisms proposed as viable explanations of the SPE, though not all are equally plausible or empirically supported (Krause, 2015b; Erdfelder \& Kroneisen, 2014; Nairne \& Pandeirada, 2016).

In their counter-argument to those who are skeptical of Darwinian influence on the SPE, Nairne and Pandeirada (2016) proposed that, just as other behavioral and physical systems co-opt existing phenotypic material for new purposes (e.g., are "exapted"), survival processing may utilize other core memory processes, such as the distinctiveness of the encoding task, to optimize memory performance. Framing the SPE in this way may preserve an evolutionary account for how episodic memory could evolve to enhance fitness (insofar as survival influences it). However, it remains puzzling that processes such as inclusive fitness (the present study) and mating success (Sandry et al., 2013) would not likewise appear to carry any novel or added mnemonic advantages. The strength upon which selection acts on 
fitness enhancing phenotypes has been evidenced in multiple domains (e.g., cognitive, biological, behavioral) - future work will hopefully provide answers as to whether memory has been influenced by these or other evolutionary processes.

\section{Acknowledgment}

We are grateful to James Gilkinson for assistance with database and analysis protocol.

\section{References}

Archer, J. (2013). Can evolutionary principles explain patterns of family violence? Psychological Bulletin, 139, 403440.

Boakes, R. (1984). From Darwin to behaviourism. Cambridge, UK: Cambridge University Press.

Cunningham, S. J., Brady-Van den Bos, M., Gill, L., \& Turk, D. J. (2013). Survival of the selfish: Contrasting selfreferential and survival-based encoding. Consciousness and Cognition, 22, 237-244.

Domjan, M., Cusato, B., \& Krause, M. (2004). Learning with arbitrary versus ecological conditioned stimuli: Evidence from sexual conditioning. Psychonomic Bulletin \& Review, 11, 232-246.

Domjan, M., \& Galef, B. G. (1983). Biological constraints on instrumental and classical conditioning: Retrospect and prospect. Animal Learning and Behavior, 11, 151-161.

Domjan, M., Mahometa, M. J., \& Matthews, R. N. (2012). Learning in intimate connections: Conditioned fertility and its role in sexual competition. Socioaffective Neuroscience and Psychology, 2, 1-10.

Dunlap, A. S., \& Stephens, D. W. (2014). Experimental evolution of prepared learning. Proceedings of the National Academy of Sciences USA, 111, 11750-11755.

Erdfelder, E., \& Kroneisen, M. (2014). Proximate cognitive mechanisms underlying the survival processing effect. In B. L. Schwartz, M. L. Howe, M. P. Toglia, \& H. Otgar (Eds.), What is adaptive about adaptive memory? (pp. 172-198). New York: Oxford University Press.

Garcia, J., \& Koelling, R. A. (1966). Relation of cue to consequence in aversion learning. Psychonomic Science, 4, 123-124.

Gould, K. L., Gilbertson, K. E., Hrvol, A. J., Nelson, J. C., Seyfer, A. L., Brantner, R. M., \& Kamil, A. C. (2013). Differences in relative hippocampus volume and number of hippocampus neurons among five corvid species. Brain, Behavior, and Evolution, 81, 56-70.

Hamilton, W. D. (1964). The genetical evolution of social behavior. I. Journal of Theoretical Biology, 7, 1-16.

Haskett, A., Skou, K., Krause, M. A., \& Taylor, J. T. (2014, May). Future tense perspective and the survival processing effect. Poster presented at the $26^{\text {th }}$ annual convention of the Association for Psychological Science, San Francisco, CA.

Hollis, K. L. (1997). Contemporary research on Pavlovian conditioning: A "new" functional analysis. American Psychologist, 52, 956-965.

Howe, M. L., \& Otgaar, H. (2013). Proximate mechanisms and the development of adaptive memory. Current Directions in Psychological Science, 22, 16-22.

Isbell, L. A. (2006). Snakes as agents of evolutionary change in primate brains. Journal of Human Evolution, 51, 135.

Kang, S. H. K., McDermott, K. B., \& Cohen, S. M. (2008). The mnemonic advantage of processing fitness-relevant information. Memory and Cognition, 36, 1151-1156.

Kazanas, S. A., \& Altarriba, J. (2015). The survival advantage: Underlying mechanisms and extant limitations. Evolutionary Psychology, 13, 360-396.

Klein, S. B. (2012). A role for self-referential processing in tasks requiring participants to imagine survival on the savannah. Journal of Experimental Psychology: Learning, Memory, and Cognition, 38, 1234-1242.

Klein, S. B., Robertson, T. E., \& Delton, A. W. (2010). Facing the future: Memory as an evolved system for planning future acts. Memory \& Cognition, 38, 13-22.

Klein, S. B., Robertson, T. E., \& Delton, A. W. (2011). The future-orientation of memory: Planning as a key component mediating the high levels of recall found with survival processing. Memory, 19, 121-139.

Kostic, B., McFarlan, C. C., \& Cleary, A. M. (2012). Extensions of the survival advantage in memory: Examining the role of ancestral context and implied social isolation. Journal of Experimental Psychology: Learning, Memory, and Cognition, 38, 1091-1098. 
Krause, M. A. (2015a). Evolutionary perspectives on learning: Conceptual and methodological issues in the study of adaptive specializations. Animal Cognition, 18, 807-820.

Krause, M. A. (2015b). Adaptive memory in humans from a comparative perspective. International Journal of Comparative Psychology, 28. (special issue on biological constraints on learning, http://escholarship.org/uc/uclapsych_ijcp)

Krause, M. A, \& Domjan, M. (2017). Ethological and evolutionary perspectives on Pavlovian conditioning. In J. Call (Ed.), Handbook of comparative psychology: Vol 2: Perception, learning and cognition (pp. 247-266). Washington, DC: American Psychological Association.

Kroneisen, M., \& Erdfelder, E. (2011). On the plasticity of the survival processing effect. Journal of Experimental Psychology: Learning, Memory and Cognition, 37, 1552-1563.

Logue, A. W. (1979). Taste aversion and the generality of the laws of learning. Psychological Bulletin, 86, 276-296.

Mills, C., \& D'Mello, S. (2014). On the validity of the autobiographical emotional memory task for emotion induction. PloS One, 9(4), e95837.

Müller, S., \& Renkewitz, F. (2015). Replication of Study 2 by Nairne, J. S., Pandeirada, J. N. S., \& Thompson, S. (Psychological Science, 2008). Open Science Framework: Reproducibility Project. Retrieved from https://osf.io/v4d2b/?action=download\&version=1

Nairne, J. S., \& Pandeirada, J. N. S. (2008). Adaptive memory: Remembering with a stone-age brain. Current Directions in Psychological Science, 17, 239-243.

Nairne, J. S., \& Pandeirada, J. N. S. (2016). Adaptive memory: The evolutionary significance of survival processing. Perspectives on Psychological Science, 11, 496-511.

Nairne, J. S., Thompson, S. R., \& Pandeirada, J. N. S. (2007). Adaptive memory: Survival processing enhances retention. Journal of Experimental Psychology: Learning, Memory, \& Cognition, 33, 263-273.

Nowack, M. A., McAvoy, A., Allen, B., \& Wilson, E. O. (2017). The general form of Hamilton's rule makes no predictions and cannot be tested empirically. Proceedings of the National Academy of Sciences, 114, 56655670.

Öhman, A., \& Mineka, S. (2001). Fears, phobias, and preparedness: Toward an evolved module of fear and fear learning. Psychological Review, 108(, 483-522.

Palmore, C. C., Garcia, A. D., Bacon, L. P., Johnson, C. A., \& Kelemen, W. L. (2012). Congruity influences memory and judgments of learning during survival processing. Psychonomic Bulletin and Review, 19, 119125.

Pandeirada, J. N. S., Fernandes, N. L., Vasconcelos, M., \& Nairne, J. S. (2017). Adaptive memory: Remembering potential mates. Evolutionary Psychology, 15, 1-11.

Park, J. H. (2007). Persistent misunderstandings of inclusive fitness and kin selection: Their ubiquitous appearance in social psychology textbooks. Evolutionary Psychology, 5, 860-873.

Parr, L. (2011). The evolution of face processing in primates. Philosophical Transactions of the Royal Society B, $366,1764-1777$.

Rand, D. G., \& Nowak, M. A. (2013). Human cooperation. Trends in Cognitive Sciences, 17, 413-425.

Sandry, J., Trafimow, D., Marks, M. J., \& Rice, S. (2013). Adaptive memory: Evaluating alternative forms of fitness-relevant processing in the survival processing paradigm. PLOS One, 8, e60868.

Savine, A. C., Scullin, M. K., Roediger III, H. L. (2011). Survival processing of faces. Memory and Cognition, 39, 1359-1373.

Scofield, J. E., Buchanan, E. M., \& Kostic, B. (2018). A meta-analysis of the survival-processing advantage in memory. Psychonomic Bulletin \& Review, 25, 997-1012.

Shier, D., \& Owings, D. (2006). Effects of predator training on behavior and post-release survival of captive prairie dogs (Cynomys ludovicianus). Biological Conservation, 132, 126-135.

Symons, C. S., \& Johnson, B. T. (1997). The self-reference effect in memory: A meta-analysis. Psychological Bulletin, 121, 371-394.

Schwartz, B. L., Howe, M. L., Toglia, M. P., \& Otgaar, H. (2014). What is adaptive about adaptive memory? New York: Oxford University Press.

Soderstrom, N. C, \& Cleary, A. M. (2014). On the domain-specificity of survival processing advantages in memory. In B. L. Schwartz, M. L. Howe, M P. Toglia, \& H. Otgaar (Eds.), What is adaptive about adaptive memory? (pp. 110-122). Oxford: Oxford University Press.

Soderstrom, N. C., \& McCabe, D. P. (2011). Are survival processing memory advantages based on ancestral priorities? Psychonomic Bulletin \& Review, 18, 564-569. 
Taylor, J., Krause, M., Rosewood, M., Petrovich, S., Doyle, T., \& Rosen, J. (2013, April). The survival memory advantage is not a deep processing effect. Poster presented at the 93rd meeting of the Western Psychological Association, Reno, NV. 